\title{
Type la supernovae: exposing models to observations
}

\section{F. K. Röpke ${ }^{*}{ }^{a}$ M. Fink, ${ }^{a}$ W. Hillebrandt, ${ }^{b}$ M. Kromer,${ }^{b}$ R. Pakmor,,${ }^{c}$ A. J. Ruiter, ${ }^{b}$}

\section{R. Seitenzahl, ${ }^{a}$ and S. A. Sim ${ }^{d}$}

${ }^{a}$ Institut für Theoretische Physik und Astrophysik, Universität Würzburg, Emil-Fischer-Straße 31, 97074 Würzburg, Germany

${ }^{b}$ Max-Planck-Institut für Astrophysik, Karl-Schwarzschild-Straße 1, 85748 Garching, Germany

${ }^{c}$ Heidelberger Institut für Theoretische Studien, Schloss-Wolfsbrunnenweg 35, D-69118 Heidelberg, Germany

${ }^{d}$ Research School of Astronomy and Astrophysics, Mount Stromlo Observatory, Cotter Road, Weston Creek, ACT 2611, Australia

E-mail: friedrich.roepkedastro.uni-wuerzburg.de, mfinkeastro.uni-wuerzburg.de, wfh@mpa-garching.mpg.de, mkromer@mpa-garching.mpg.de, ruediger.pakmor@h-its.org, ajr@mpa-garching.mpg.de, iseitenzahleastro.uni-wuerzburg.de, ssimemso.anu. edu. au

Type Ia supernovae play an important role in observational cosmology and in various astrophysical processes. As main producers of iron group elements, they are a key component in the cosmic cycle of matter. The questions about the nature of the progenitor systems from which these cosmic explosions arise and of their physical mechanism, however, are not settled. Several possibilities have been suggested in the literature. We discuss recent developments and present multidimensional explosion simulations referring to two different scenarios. Combined with detailed radiative transfer calculations that predict observables from the models and allow direct comparison with astronomical data, these simulations shed light on the nature of the progenitor systems. Although the range of observed properties is large enough to accommodate many theoretical explosion models, it is particularly important to identify the class of objects that constitutes the bulk of Type Ia supernovae. Even when starting out from systems as diverse as Chandrasekhar-mass white dwarfs and double white dwarf mergers, there is some degeneracy in the predicted observables that prevents a straightforward judgement on the validity of the models. We discuss possible ways to overcome this problem and to discriminate between different scenarios.

XII International Symposium on Nuclei in the Cosmos

August 5-12, 2012

Cairns, Australia

${ }^{*}$ Speaker. 


\section{Introduction}

Apart from their prominent role in observational cosmology [1, 2], Type Ia supernovae (SNe Ia) are important in many astrophysical processes. One of these is the cosmic cycle of matter. With the products of explosive nucleosynthesis ejected into the interstellar material, they are the main contributor to iron group elements in the Universe, but also have some share in the enrichment of intermediate-mass elements (most importantly, $\mathrm{Si}$ to $\mathrm{Ca}$ ) and may be responsible for about half of the p-process elements (see contribution of C. Travaglio et al. in this volume and [3]).

With their bright optical display, they are one of the drivers of current surveys searching for astrophysical transients. Observationally, the class of SNe Ia is well-studied, but it is only partially understood theoretically. The astronomical classification is according to spectral features. Unlike other supernovae, $\mathrm{SNe}$ Ia do not show hydrogen and helium lines, but a prominent silicon line is visible around the time of maximum $B$-band luminosity. Other typical features of $\mathrm{SNe}$ Ia spectra result from intermediate-mass elements (e.g., sulfur) and iron group elements.

\section{Physical mechanism of SNe Ia and explosion scenarios}

The greatest obstacle of modeling SNe Ia theoretically is their unknown progenitor system. Although they are associated with thermonuclear explosions of carbon-oxygen white dwarfs [4, 5], it is unclear what drives the star to ignition.

Setting aside this question for the moment, several conclusions can be drawn from the notion of thermonuclear combustion in degenerate carbon-oxygen material already. As burning begins with ${ }^{12} \mathrm{C}+{ }^{12} \mathrm{C}$ fusion, a reaction whose reaction rate scales with a high power of temperature, burning must be confined to a thin front. The internal width of such a combustion front is only of the order of millimeters to centimeters and thus much smaller than the scales of the white dwarf star (with a radius of $\sim 2,000 \mathrm{~km}$ for a white dwarf close to Chandrasekhar mass). It can therefore be described as a sharp discontinuity between nuclear fuel and nuclear ash. The conservation laws of hydrodynamics allow for two distinct modes of propagation for such combustion waves: subsonic deflagrations and supersonic detonations. In both cases, the composition of the ash (and thus also the nuclear energy release) depends to first order on the fuel density ahead of the combustion wave. At the highest densities, as encountered at the center of white dwarfs, nuclear statistical equilibrium is reached and ultimately iron group elements are produced in the freeze-out from this equilibrium. At lower densities, burning becomes incomplete and intermediate-mass elements (such as $\mathrm{Si}, \mathrm{S}$, and $\mathrm{Ca}$ ) are synthesized. At even lower densities, oxygen fusion ceases and only carbon burning remains active. Below a certain density threshold the combustion extinguishes completely and the fuel material is left unchanged. This picture agrees with the characteristic spectral features of SNe Ia and hence, in turn, the spectra of these events can be regarded as prototypical for a thermonuclear explosion. Starting out burning from predominantly self-conjugate matter $\left({ }^{12} \mathrm{C}\right.$ and ${ }^{16} \mathrm{O}$ ), the most abundant iron group nuclide in the ashes is ${ }^{56} \mathrm{Ni}$. This explains $[6,7]$ why $\mathrm{SNe}$ Ia are extremely bright - and stay bright and observable for weeks and months. The radioactive decay of ${ }^{56} \mathrm{Ni}$ over ${ }^{56} \mathrm{Co}$ to ${ }^{56} \mathrm{Fe}$ releases gamma quanta and positrons which heat the ejecta and give rise to the emission of radiation predominantly in the optical bands (see also [8]). 
In summary, this gives a convincing picture of how SNe Ia can arise from thermonuclear explosions of carbon-oxygen white dwarfs, but it leaves open the question of why it would explode in the first place. Several possibilities have been suggested (see [9] for an overview) and traditionally the progenitors in question are classified into single-degenerate systems, in which the white dwarf accretes matter from a normal companion star, and double-degenerate systems, in which the supernova arises from the interaction between two white dwarfs.

There is no one-to-one correspondence between progenitor systems and explosion scenarios. Whereas white dwarf explosions when approaching the Chandrasekhar mass are usually associated with the single-degenerate channel, sub-Chandrasekhar mass explosions may arise in single and double degenerate systems. In double-degenerate systems, of course, the mass of the merged and exploding object can also exceed the Chandrasekhar mass, thus formally resulting in superChandrasekhar mass explosions [10].

Since the question of the progenitors of SNe Ia is open and, according to observations, there are different sub-classes of SNe Ia, several scenarios could contribute to the sample. About $70 \%$ of all SNe Ia are normal objects [11] - so which scenario explains these events? For answering such questions it is important to construct consistent hydrodynamical explosion models and to compare the observables predicted from them to astronomical data. A possibility for this is a modeling pipeline that captures the explosion dynamics in two- or three-dimensional simulations (e.g., $[12,13,14,15,16,17])$ followed by a postprocessing step $[18,19,20,21]$ to recover the detailed nucleosynthesis of the explosion. In the resulting ejecta structure, radiation transport from the decay of radioactive species is calculated, allowing to compute synthetic observables that can then be compared to SN Ia observations. In this approach, several models have been studied. In the following, two scenarios are discussed that are promising candidates for explaining normal $\mathrm{SNe}$ Ia (see, however, $[22,23,24]$ and the contribution of M. Fink et al. in this volume for recent studies of scenarios not discussed here).

\subsection{The Chandrasekhar-mass story}

Chandrasekhar-mass explosions have to start out as subsonic deflagrations. Pure detonations are incompatible with observations as they burn the entire white dwarf at the initial high densities. This produces almost exclusively iron group elements, which is at odds with the observed spectra. Deflagrations are slow if the flame remains laminar, but buoyancy-driven instabilities wrinkle the flame on large scales and generate turbulence by secondary shear instabilities (e.g., [25]). If the burning stays subsonic throughout the explosion, the result reaches only the lower end of the brightness distribution of normal SN Ia [26]. It is, however, possible to reproduce SN 2002cx-like objects with asymmetrically ignited pure deflagration models [27].

To reach the range of brightness (and corresponding ${ }^{56} \mathrm{Ni}$ production) of normal $\mathrm{SNe}$ Ia, it is necessary to boost the burning efficiency in late stages of the explosion. A deflagration-todetonation transition offers this possibility [28, 29]. Whether or not such transitions can be spontaneously initiated in SNe Ia is still not known, but if so, strong turbulent velocity fluctuations are certainly needed [30, 31]. Three-dimensional simulations of deflagrations in white dwarfs indicate such strong turbulent velocity fluctuations, even at late stages of the burning [26].

Multi-dimensional delayed detonation simulations [15, 32, 33] produce an ejecta structure that differs significantly from that of their one-dimensional counterparts. Buoyancy carries the neutron- 
rich iron group nuclei produced at high densities near the center of the white dwarf out to larger radii. At a later stage, when the star has expanded considerably, deflagration-to-detonation transitions may initiate detonation fronts that burn out large parts of the remaining fuel. Although the outward burning in the outer layers at lower densities produces mainly intermediate-mass elements and oxygen, thus producing a chemically stratified structure, the detonation also burns downward to the center of the white dwarf in funnels and cavities of fuel in between the ash plumes of the deflagration stage. Near the center, the densities are, in large parts, still high enough for the detonation to produce iron group elements. The temperatures, however, are too low to allow efficient electron captures. Thus, in multi-dimensional delayed detonation models, the center of the ejecta is usually dominated by ${ }^{56} \mathrm{Ni}$, whereas stable iron group nuclei are found at larger radii near the transition from the iron-group dominated central region of the ejecta to the intermediate-mass elements at higher velocities. The multi-dimensional representation of the ignition configuration (e.g., [34]) allows for a substantial variation in the brightness of the modeled event [35, 36, 32, 33]. Several other parameters, however, may also contribute to the variability [37, 38, 39, 40].

Although synthetic observables derived from two-dimensional simulations reproduce the main characteristics of observed SNe Ia [41] and even - to a reasonable degree - the width-luminosity relation [32] used to calibrate SNe Ia as cosmological distance indicators [42, 43], the Chandrasekharmass model is afflicted with various problems. There are indications that the stable iron-group elements at high velocities in the ejecta may cause a reddening of the spectra that is in conflict with the observations [44]. Observations [45] and some population synthesis calculations [46] suggest that there exist too few progenitor systems to explain the observed rate of SNe Ia within the single-degenerate progenitor scenario. Moreover, the impact of the supernova blast wave on the companion star should strip off hydrogen-rich material from its surface and mix it into the ejecta $[47,48,49,50]$. No observational evidence for this material exists and some model predictions are even in contradiction to the observations. As the companion star is not destroyed in the interaction with the supernova, searches for it in historic SN Ia remnants have been carried out [51]. These, however, did not yield a convincing identification [52]. All this leads to the conclusion that alternative models have to be seriously considered. Some support for the single-degenerate progenitor scenario, however, is provided by the observed interaction of several supernovae with circumstellar material that possibly results from preceding nova eruptions (e.g., [53, 54, 55]). This has been interpreted as symbiotic nova progenitors for certain SNe Ia.

\subsection{White dwarf mergers}

A promising alternative to the Chandrasekhar-mass delayed detonation model is the merger of two carbon-oxygen white dwarf stars. The inspiral and merger was followed with a modification [56] of the cosmological smoothed particle hydrodynamics code GADGET. If the stars are massive enough and do not differ too much in their masses, a carbon detonation is likely to trigger in the merger phase at the interface between the merging stars [10]. In this merger, the more massive star is little affected by tidal interaction. Since it has the highest density in its central material, the ${ }^{56} \mathrm{Ni}$ production and thus the brightness of the event is determined by its mass. For a merger of a $1.1 \mathrm{M}_{\odot}$ white dwarf with an $0.9 \mathrm{M}_{\odot}$ companion, the predicted brightness and other observables match that of normal SNe Ia very well [57]. In principle, such violent mergers have the potential to explain a large part of the SN Ia sample. Their predicted occurrence rates may be close to the observed 
SN Ia rate and also the distribution of brightnesses may match the observations [58]. Their success in reproducing SN Ia observations is a shift in paradigm. It demonstrates that for a viable model, the explosion of the white dwarf is not necessarily bound to the Chandrasekhar mass. Moreover, the merger model removes in a natural way the complications that arise from the missing observational identification of a companion star in the single-degenerate model.

\section{SN 2011fe}

SN 2011fe is the closest Type Ia supernova observed in the last 25 years. It was detected extremely early [4], which allowed to convincingly identify the exploding object as a white dwarf $[4,5]$. Archival data [59, 60] and observations in the UV [61] and radio [62, 63] bands put strong constraints on a non-degenerate companion star.

A comparison with simulations and predicted observables of a delayed detonation in a Chandrasekhar-mass white dwarf and a violent merger (both reaching the brightness of SN 2011fe) showed that it is difficult to give preference to one of these models based on optical observations near peak luminosity [44]. To break the degeneracy, other observations are required.

The nucleosynthesis in the two scenarios proceeds under very different conditions. In the delayed detonation Chandrasekhar-mass model, iron group elements are produced at high densities and due to normal freeze-out from nuclear statistical equilibrium. In contrast, in the merger model, densities are lower and thus incomplete Si burning and alpha-rich freeze-out from nuclear statistical equilibrium produce iron group elements. This leads to different abundances of long-lived radioactive nuclides (in particular, ${ }^{57} \mathrm{Co}$ and ${ }^{55} \mathrm{Fe}$ ), which, by leptonic decay, are predicted to dominate the light curve after $\sim 900$ days. Thus, late light curves are sensitive to the iron group nucleosynthesis [64] and this may be a way of discriminating between explosion models [44]. Other possibilities include observations in X-rays [65] or gamma rays (e.g., [66]).

\section{Summary}

Early-time observations of SN 2011fe gave strong support for associating Type Ia supernovae to explosions of white dwarf stars. As the significance of this class of supernovae for astrophysics is far-reaching, ranging from observational cosmology (see, e.g., [67] for a recent study) to galactic chemical evolution, a deeper theoretical understanding is called for. This is complicated by the lack of reliable initial conditions for explosion models: the nature of the progenitor system is observationally not yet established. Two explosion scenarios that arise from different possibilities and that hold promise for explaining normal SNe Ia have been presented: delayed detonations in Chandrasekhar-mass white dwarfs and violent mergers of white dwarfs. At the moment, however, it is hard to distinguish them on the basis of optical observables near peak brightness. Alternatives that are more sensitive to details of the isotopic composition of the ejecta have been discussed.

\section{Questions from the audience}

Q. Peng: "How do you think whether SN Ia may be taken as a standard candle?" 
Answer: A calibration is certainly necessary to use them as distance indicators. The relations that are currently used are purely empirical but nonetheless very successful. With improved modeling, we hope to be able to deliver a sound theoretical basis for the use of SNe Ia as cosmic distance indicators.

A. Heger: "Would the scenario of a Ia delayed by rotation (spin-up-spin-down) be consistent with the non-observation of the companion star?"

Answer: This model is constructed to resolve the problem of the missing companion star and leaves behind only the core of a giant. Stripping off large amounts of material from it is hardly possible. Whether or not it should be observable as a peculiar star in the center of the remnant depends on its thermal evolution after the supernova impact which is difficult to model reliably.

H. Stoecker: "Suggestion: drop the 'perhaps' on your concluding transparency."

Answer: This referred to a cautious formulation in the assessment of progenitor channels for SNe Ia. It was stated that 'perhaps' there are not enough progenitor systems for the single-degenerate channel to explain the rate of SNe Ia. This is still controversial. Since modeling the progenitor system is a challenging problem of binary stellar evolution and since population synthesis calculations have many uncertainties, definite conclusions can unfortunately not be drawn yet.

L. Nittler: "What happens if two O-Ne white dwarfs merge?"

Answer: This is an interesting problem. From accretion-induced collapse simulations of the 1990s it seems likely that such a merger could lead to a gravitational collapse towards a neutron star rather than a thermonuclear explosion. Detonations in O-Ne white dwarf matter, however, may result in the opposite. Thus it is worthwhile exploring whether such detonations can be triggered in O-Ne white dwarf mergers.

B. Schmidt: "One way to overcome problems with using a degenerate scenario would be to have explosion delayed by a Gyr due to rotation of WD. What are the effects of a rapidly rotating $>1.38 \mathrm{M}_{\odot}$ WD on explosions?"

Answer: A rapid differential rotation (which is required to increase the mass of the white dwarf significantly) leads to problems in the delayed detonation scenario. Buoyancy instabilities in the deflagration phase are suppressed in the equatorial direction due to angular momentum conservation. Therefore, the deflagration is not very efficient in pre-expanding the white dwarf before the detonation triggers. This inevitably leads to very bright events that in their predicted observables are inconsistent with normal SNe Ia. A rigid rotation, however, does probably not affect the explosion very much, but this has yet to be confirmed in simulations.

\section{Acknowledgments}

This work was supported by the Deutsche Forschungsgemeinschaft via the Emmy Noether Program (RO 3676/1-1) and by the ARCHES prize of the German Federal Ministry of Edu- 
cation and Research (BMBF). Funding for German-Australian collaboration was provided by a DAAD/Go8 grant. FKR thanks the organizers of NIC for financial support.

\section{References}

[1] A. G. Riess, A. V. Filippenko, P. Challis, A. Clocchiatti, A. Diercks et al. Observational Evidence from Supernovae for an Accelerating Universe and a Cosmological Constant. AJ, 116 (1998) 1009. [arXiv:astro-ph/9805201].

[2] S. Perlmutter, G. Aldering, G. Goldhaber, R. A. Knop, P. Nugent et al. Measurements of Omega and Lambda from 42 High-Redshift Supernovae. ApJ, 517 (1999) 565.

[arXiv:astro-ph/9812133].

[3] C. Travaglio, F. K. Röpke, R. Gallino and W. Hillebrandt. Type Ia Supernovae as Sites of the p-process: Two-dimensional Models Coupled to Nucleosynthesis. ApJ, 739 (2011) 93. [1106.0582].

[4] P. E. Nugent, M. Sullivan, S. B. Cenko, R. C. Thomas, D. Kasen et al. Supernova SN 2011 fe from an exploding carbon-oxygen white dwarf star. Nature, $\mathbf{4 8 0}$ (2011) 344. [1110.6201] .

[5] J. S. Bloom, D. Kasen, K. J. Shen, P. E. Nugent, N. R. Butler et al. A Compact Degenerate Primary-star Progenitor of SN 2011fe. ApJ, 744 (2012) L17. [1111.0966].

[6] J. W. Truran, W. D. Arnett and A. G. W. Cameron. Nucleosynthesis in supernova shock waves. Canadian Journal of Physics, 45 (1967) 2315.

[7] S. A. Colgate and C. McKee. Early Supernova Luminosity. ApJ, 157 (1969) 623.

[8] M. J. Kuchner, R. P. Kirshner, P. A. Pinto and B. Leibundgut. Evidence for ${ }^{56} \mathrm{Ni} \rightarrow{ }^{56} \mathrm{Co} \rightarrow{ }^{56} \mathrm{Fe}$ decay in type Ia supernovae. ApJ, 426 (1994) L89.

[9] W. Hillebrandt and J. C. Niemeyer. Type Ia Supernova Explosion Models. ARA\&A, 38 (2000) 191. [arXiv:astro-ph/0006305].

[10] R. Pakmor, M. Kromer, F. K. Röpke, S. A. Sim, A. J. Ruiter et al. Sub-luminous type Ia supernovae from the mergers of equal-mass white dwarfs with mass $\sim 0.9 M_{\odot}$. Nature, 463 (2010) 61. [0911.0926].

[11] W. Li, J. Leaman, R. Chornock, A. V. Filippenko, D. Poznanski et al. Nearby supernova rates from the Lick Observatory Supernova Search - II. The observed luminosity functions and fractions of supernovae in a complete sample. MNRAS, 412 (2011) 1441. [1006.4612] .

[12] F. K. Röpke and W. Hillebrandt. Full-star type Ia supernova explosion models. A\&A, 431 (2005) 635. [arXiv:astro-ph/0409286].

[13] F. K. Röpke. Following multi-dimensional type Ia supernova explosion models to homologous expansion. A\&A, 432 (2005) 969. [arXiv:astro-ph/ 0408296 ] .

[14] S. I. Blinnikov, F. K. Röpke, E. I. Sorokina, M. Gieseler, M. Reinecke et al. Theoretical light curves for deflagration models of type Ia supernova. A\&A, 453 (2006) 229.

[arXiv:astro-ph/0603036].

[15] F. K. Röpke and J. C. Niemeyer. Delayed detonations in full-star models of type Ia supernova explosions. A\&A, 464 (2007) 683. [arXiv: astro-ph/0703378] . 
[16] W. Hillebrandt, S. A. Sim and F. K. Röpke. Off-center explosions of Chandrasekhar-mass white dwarfs: an explanation of super-bright type Ia supernovae? A\&A, 465 (2007) L17. [arXiv:astro-ph/0702344].

[17] S. A. Sim, D. N. Sauer, F. K. Röpke and W. Hillebrandt. Light curves for off-centre ignition models of Type Ia supernovae. MNRAS, 378 (2007) 2. [arXiv: astro-ph/0 703764 ] .

[18] C. Travaglio, W. Hillebrandt, M. Reinecke and F.-K. Thielemann. Nucleosynthesis in multi-dimensional SN Ia explosions. A\&A, 425 (2004) 1029. [arXiv: astro-ph/0 06281 ] .

[19] F. K. Röpke, M. Gieseler, M. Reinecke, C. Travaglio and W. Hillebrandt. Type Ia supernova diversity in three-dimensional models. A\&A, 453 (2006) 203. [arXiv:astro-ph/0 506107 ] .

[20] I. R. Seitenzahl, F. K. Röpke, M. Fink and R. Pakmor. Nucleosynthesis in thermonuclear supernovae with tracers: convergence and variable mass particles. MNRAS, 407 (2010) 2297. [1 005.5071 ] .

[21] K. Maeda, F. K. Röpke, M. Fink, W. Hillebrandt, C. Travaglio et al. Nucleosynthesis in Two-Dimensional Delayed Detonation Models of Type Ia Supernova Explosions. ApJ, 712 (2010) 624. [1002.2153].

[22] M. Fink, W. Hillebrandt and F. K. Röpke. Double-detonation supernovae of sub-Chandrasekhar mass white dwarfs. A\&A, 476 (2007) 1133. [arXiv:0710.5486].

[23] M. Fink, F. K. Röpke, W. Hillebrandt, I. R. Seitenzahl, S. A. Sim et al. Double-detonation sub-Chandrasekhar supernovae: can minimum helium shell masses detonate the core? A\&A, $\mathbf{5 1 4}$ (2010) A53. [1002.2173].

[24] M. Kromer, S. A. Sim, M. Fink, F. K. Röpke, I. R. Seitenzahl et al. Double-detonation Sub-Chandrasekhar Supernovae: Synthetic Observables for Minimum Helium Shell Mass Models. ApJ, 719 (2010) 1067. [1006.4489].

[25] F. K. Röpke and W. Schmidt. Turbulent combustion in thermonuclear supernovae. In W. Hillebrandt and F. Kupka, eds., Interdisciplinary Aspects of Turbulence, Lecture Notes in Physics, pp. 255-289. Springer-Verlag, Berlin, 2009. [arXiv : ?? ?]

[26] F. K. Röpke. Flame-driven Deflagration-to-Detonation Transitions in Type Ia Supernovae? ApJ, 668 (2007) 1103. [arXiv:0709.4095].

[27] M. Kromer, M. Fink, V. Stanishev, S. Taubenberger, F. Ciaraldi-Schoolman et al. 3D deflagration simulations leaving bound remnants: a model for 2002cx-like Type Ia supernovae. MNRAS, (2012) 428. [1210.5243].

[28] S. I. Blinnikov and A. M. Khokhlov. Development of Detonations in Degenerate Stars. Soviet Astronomy Letters, 12 (1986) 131.

[29] A. M. Khokhlov. Delayed detonation model for type Ia supernovae. A\&A, 245 (1991) 114.

[30] S. E. Woosley. Type Ia Supernovae: Burning and Detonation in the Distributed Regime. ApJ, 668 (2007) 1109. [arXiv:0709.4237].

[31] S. E. Woosley, A. R. Kerstein, V. Sankaran, A. J. Aspden and F. K. Röpke. Type Ia Supernovae: Calculations of Turbulent Flames Using the Linear Eddy Model. ApJ, 704 (2009) 255. [0811.3610].

[32] D. Kasen, F. K. Röpke and S. E. Woosley. The diversity of type Ia supernovae from broken symmetries. Nature, 460 (2009) 869. [0907.0708] . 
[33] I. R. Seitenzahl, F. Ciaraldi-Schoolmann, F. K. Röpke, M. Fink, W. Hillebrandt et al. Three-dimensional delayed-detonation models with nucleosynthesis for Type Ia supernovae. MNRAS, (2012) 315. [1211.3015] .

[34] F. K. Röpke, W. Hillebrandt, J. C. Niemeyer and S. E. Woosley. Multi-spot ignition in type Ia supernova models. A\&A, 448 (2006) 1. [arXiv: astro-ph/0510474].

[35] F. K. Röpke, S. E. Woosley and W. Hillebrandt. Off-Center Ignition in Type Ia Supernovae. I. Initial Evolution and Implications for Delayed Detonation. ApJ, 660 (2007) 1344. [arXiv:astro-ph/0609088].

[36] P. A. Mazzali, F. K. Röpke, S. Benetti and W. Hillebrandt. A Common Explosion Mechanism for Type Ia Supernovae. Science, 315 (2007) 825. [arXiv : astro-ph/0702351] .

[37] I. Seitenzahl. Internal conversion electrons and supernova light curves. Progress in Particle and Nuclear Physics, 66 (2011) 329 . Particle and Nuclear Astrophysics, International Workshop on Nuclear Physics, 32nd Course.

[38] D. M. Townsley, A. P. Jackson, A. C. Calder, D. A. Chamulak, E. F. Brown et al. Evaluating Systematic Dependencies of Type Ia Supernovae: The Influence of Progenitor ${ }^{22} \mathrm{Ne}$ Content on Dynamics. ApJ, 701 (2009) 1582. [0906.4384].

[39] A. P. Jackson, A. C. Calder, D. M. Townsley, D. A. Chamulak, E. F. Brown et al. Evaluating Systematic Dependencies of Type Ia Supernovae: The Influence of Deflagration to Detonation Density. ApJ, 720 (2010) 99. [1007.1138].

[40] B. K. Krueger, A. P. Jackson, D. M. Townsley, A. C. Calder, E. F. Brown et al. On Variations of the Brightness of Type Ia Supernovae with the Age of the Host Stellar Population. ApJ, 719 (2010) L5. [1007.0910].

[41] S. Blondin, D. Kasen, F. K. Röpke, R. P. Kirshner and K. S. Mandel. Confronting 2D delayed-detonation models with light curves and spectra of Type Ia supernovae. MNRAS, 417 (2011) 1280. [1107.0009].

[42] M. M. Phillips. The absolute magnitudes of Type Ia supernovae. ApJ, 413 (1993) L105.

[43] M. M. Phillips, P. Lira, N. B. Suntzeff, R. A. Schommer, M. Hamuy et al. The Reddening-Free Decline Rate Versus Luminosity Relationship for Type Ia Supernovae. AJ, 118 (1999) 1766. [arXiv:astro-ph/9907052].

[44] F. K. Röpke, M. Kromer, I. R. Seitenzahl, R. Pakmor, S. A. Sim et al. Constraining Type Ia Supernova Models: SN 201 lfe as a Test Case. ApJ, 750 (2012) L19. [1203.4839] .

[45] M. Gilfanov and Á. Bogdán. An upper limit on the contribution of accreting white dwarfs to the typeIa supernova rate. Nature, $\mathbf{4 6 3}$ (2010) 924. [1002.3359] .

[46] A. J. Ruiter, K. Belczynski and C. Fryer. Rates and Delay Times of Type Ia Supernovae. ApJ, 699 (2009) 2026. [0904.3108].

[47] E. Marietta, A. Burrows and B. Fryxell. Type Ia Supernova Explosions in Binary Systems: The Impact on the Secondary Star and Its Consequences. ApJS, 128 (2000) 615. [arXiv:astro-ph/9908116].

[48] R. Pakmor, F. K. Röpke, A. Weiss and W. Hillebrandt. The impact of type Ia supernovae on main sequence binary companions. A\&A, $\mathbf{4 8 9}$ (2008) 943. [0807.3331] . 
[49] Z. W. Liu, R. Pakmor, F. K. Röpke, P. Edelmann, B. Wang et al. Three-dimensional simulations of the interaction between Type Ia supernova ejecta and their main sequence companions. A\&A, 548 (2012) A2. [1209.4458].

[50] K.-C. Pan, P. M. Ricker and R. E. Taam. Impact of Type Ia Supernova Ejecta on Binary Companions in the Single-degenerate Scenario. ApJ, 750 (2012) 151. [1203.1932] .

[51] P. Ruiz-Lapuente, F. Comeron, J. Méndez, R. Canal, S. J. Smartt et al. The binary progenitor of Tycho Brahe's 1572 supernova. Nature, 431 (2004) 1069. [arXiv : astro-ph/ 0410673 ] .

[52] W. E. Kerzendorf, B. P. Schmidt, J. B. Laird, P. Podsiadlowski and M. S. Bessell. Hunting for the Progenitor of SN 1006: High-resolution Spectroscopic Search with the FLAMES Instrument. ApJ, 759 (2012) 7. [1207.4481].

[53] F. Patat, P. Chandra, R. Chevalier, S. Justham, P. Podsiadlowski et al. Detection of Circumstellar Material in a Normal Type Ia Supernova. Science, 317 (2007) 924. [arXiv: 0707.2793 ] .

[54] A. Sternberg, A. Gal-Yam, J. D. Simon, D. C. Leonard, R. M. Quimby et al. Circumstellar Material in Type Ia Supernovae via Sodium Absorption Features. Science, 333 (2011) 856. [11 08 . 3664 ].

[55] B. Dilday, D. A. Howell, S. B. Cenko, J. M. Silverman, P. E. Nugent et al. PTF 11kx: A Type Ia Supernova with a Symbiotic Nova Progenitor. Science, 337 (2012) 942. [1207. 1306 ] .

[56] R. Pakmor, P. Edelmann, F. K. Röpke and W. Hillebrandt. Stellar GADGET: a smoothed particle hydrodynamics code for stellar astrophysics and its application to Type Ia supernovae from white dwarf mergers. MNRAS, 424 (2012) 2222. [1205.5806].

[57] R. Pakmor, M. Kromer, S. Taubenberger, S. A. Sim, F. K. Röpke et al. Normal Type Ia Supernovae from Violent Mergers of White Dwarf Binaries. ApJ, 747 (2012) L10. [1201 . 5123] .

[58] A. J. Ruiter, S. A. Sim, R. Pakmor, M. Kromer, I. R. Seitenzahl et al. On the brightness distribution of Type Ia supernovae from violent white dwarf mergers. MNRAS, (2012) 362. [1209.0645] .

[59] W. Li, J. S. Bloom, P. Podsiadlowski, A. A. Miller, S. B. Cenko et al. Exclusion of a luminous red giant as a companion star to the progenitor of supernova SN 2011 fe. Nature, 480 (2011) 348. [1109.1593].

[60] J. Liu, R. Di Stefano, T. Wang and M. Moe. On the Nature of the Progenitor of the Type Ia SN2011fe in M101. ApJ, 749 (2012) 141. [1110.2506].

[61] P. J. Brown, K. S. Dawson, M. de Pasquale, C. Gronwall, S. Holland et al. A Swift Look at SN 2011fe: The Earliest Ultraviolet Observations of a Type Ia Supernova. ApJ, 753 (2012) 22. [1110.2538] .

[62] A. Horesh, S. R. Kulkarni, D. B. Fox, J. Carpenter, M. M. Kasliwal et al. Early Radio and X-Ray Observations of the Youngest nearby Type Ia Supernova PTF 11kly (SN 2011fe). ApJ, 746 (2012) 21. [1109.2912].

[63] L. Chomiuk, A. M. Soderberg, M. Moe, R. A. Chevalier, M. P. Rupen et al. EVLA Observations Constrain the Environment and Progenitor System of Type Ia Supernova 2011fe. ApJ, 750 (2012) 164. [1201.0994].

[64] I. R. Seitenzahl, S. Taubenberger and S. A. Sim. Late-time supernova light curves: the effect of internal conversion and Auger electrons. MNRAS, 400 (2009) 531. [0908.0247].

[65] K. Maeda, Y. Terada, D. Kasen, F. K. Röpke, A. Bamba et al. Prospect of Studying Hard X-and Gamma-Rays from Type Ia Supernovae. ApJ, 760 (2012) 54. [1208. 2094 ] . 
[66] A. Summa, A. Ulyanov, M. Kromer, S. Boyer, F. K. Röpke et al. Gamma-ray diagnostics of Type Ia supernovae: Predictions of observables from three-dimensional modeling (2012). Submitted to Astronomy and Astrophysics.

[67] S. Benitez-Herrera, F. Röpke, W. Hillebrandt, C. Mignone, M. Bartelmann et al. Model-independent reconstruction of the expansion history of the Universe from Type Ia supernovae. MNRAS, 419 (2012) 513. [1109.0873]. 